\title{
Correction to: Transcriptional inhibition by CDK7/9 inhibitor SNS-032 abrogates oncogene addiction and reduces liver metastasis in uveal melanoma
}

Jing Zhang, Shenglan Liu, Qianyun Ye and Jingxuan Pan*

Correction to: Mol Cancer 18, 140 (2019)

https://doi.org/10.1186/s12943-019-1070-7

Following publication of the original article [1], minor errors were identified in the images presented in Figs. 1, 2 and 4; specifically:

- Fig. 1b: immunoblot band for p-RNA Pol II (S2), Omm2.3 cells, has been replaced with the correct image

- Fig. 1c: immunoblot bands for p-YAP (S127), in both Mel270 and Omm2.3 cells, have been replaced with the correct images

- Fig. 2c: immunoblot band for Actin, Mel270 cells, has been replaced with the correct image

- Fig. 4d: immunoblot bands for Sox2, in both 92.1 and Mel270 cells, have been replaced with the correct images

The corrected figures are given below. The correction does not have any effect on the results or conclusions of the paper. The original article has been corrected.
Published online: 16 October 2021

Reference

1. Zhang J, Liu S, Ye Q, et al. Transcriptional inhibition by CDK7/9 inhibitor SNS-032 abrogates oncogene addiction and reduces liver metastasis in uveal melanoma. Mol Cancer. 2019;18:140. https://doi.org/10.1186/ s12943-019-1070-7.

\section{Publisher's Note}

Springer Nature remains neutral with regard to jurisdictional claims in published maps and institutional affiliations. to the material. If material is not included in the article's Creative Commons licence and your intended use is not permitted by statutory regulation or exceeds the permitted use, you will need to obtain permission directly from the copyright holder. To view a copy of this licence, visit http://creativecommons.org/licenses/by/4.0/. The Creative Commons Public Domain Dedication waiver (http://creativeco mmons.org/publicdomain/zero/1.0/) applies to the data made available in this article, unless otherwise stated in a credit line to the data. 
(See figure on next page.)

Fig. 1 SNS-032 inhibits cellular growth through blocking YAP signaling in uveal melanoma (UM) cells. a The protein levels of CDK7/9 in UM cells and ARPE-19 cells were examined by Western blot analysis. b SNS-032 inhibited CTD phosphorylation of RNA Pol II in UM cells. Cells were treated with increasing concentration of SNS-032 for $48 \mathrm{~h}$, Western blot analysis with the indicated antibodies was performed. c SNS-032 blocked YAP signaling in UM cells. Mel270 and Omm2.3 cells were treated with SNS-032 for $48 \mathrm{~h}$, the protein levels of YAP, phospho-YAP (S127) and its downstream targets CTGF and CYR61 were examined by Western blot analysis. $\mathbf{d}$ SNS-032 inhibited gene transcription of YAP. The UM cells were treated with SNS-032 for $24 \mathrm{~h}$, the mRNA levels of YAP were detected by qRT-PCR analysis. e SNS-032 suppressed YAP-mediated gene transcription. UM cells were co-transfected with Gal4BD-TEAD4, G5-luciferase reporter (contains five Gal4-binding sites), and internal control Renilla luciferase reporter constructs for $24 \mathrm{~h}$, then treated with SNS-032 for another $24 \mathrm{~h}$, followed by luciferase activity assay. $\mathbf{f}$ SNS-032 reduced mRNA levels of YAP target genes. The UM cells were treated with SNS-032 for $24 \mathrm{~h}$, qRT-PCR analysis of YAP target genes CTGF and CYR61 were performed. g SNS-032 inhibited cell viability of UM cells but not ARPE- 19 cells. The cells were incubated with increasing concentrations of SNS-032 for 72 h, cell viability was measured by MTS assay. Dose-response curves from three independent experiments are shown. Error bars represent standard deviation (SD). $\mathbf{h}$ Anchorage-independent colony growth of UM cells was dramatically inhibited by SNS-032. The UM cells pretreated with different concentrations of SNS-032 were cultured in soft agar, colony-formation ability was assessed. $\mathbf{i}$ and $\mathbf{j}$ Ectopic expression of YAP attenuated the growth inhibition effect of SNS-032. UM cells stably expressing of YAP were treated with SNS-032 $(0.5 \mu \mathrm{M})$ for the indicated durations, expression of YAP in UM cells was examined by Western blot analysis (i). The cell number was counted by trypan blue exclusion assay (j). ${ }^{*}, P<0.05 ;{ }^{* *}, P<0.01$; ***, $P<0.001$, one-way ANOVA, post hoc intergroup comparisons, Tukey's test 
A

B
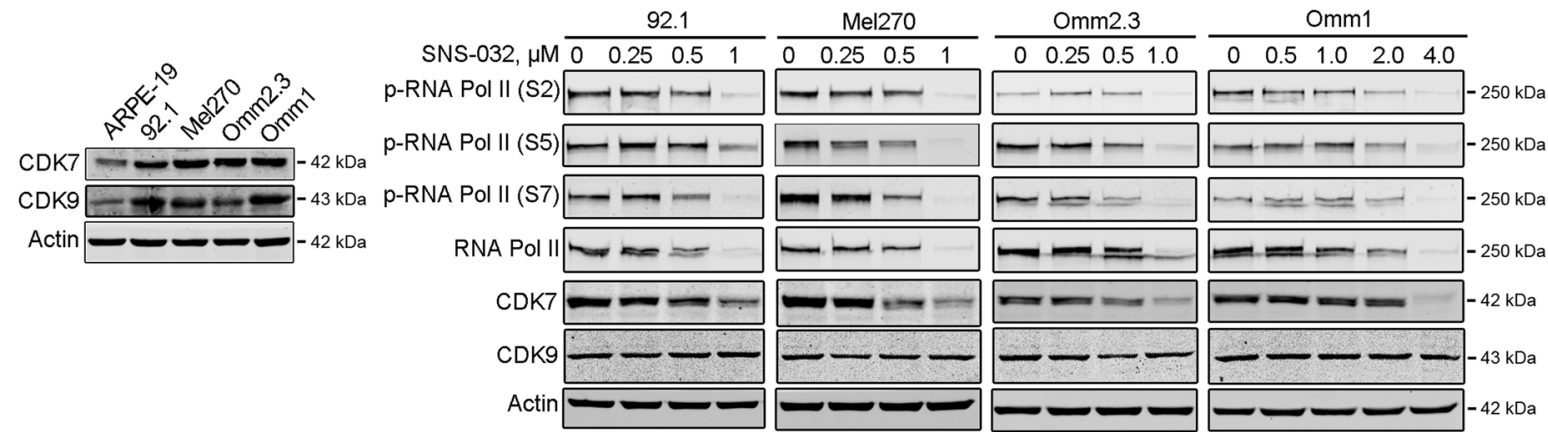

C

D
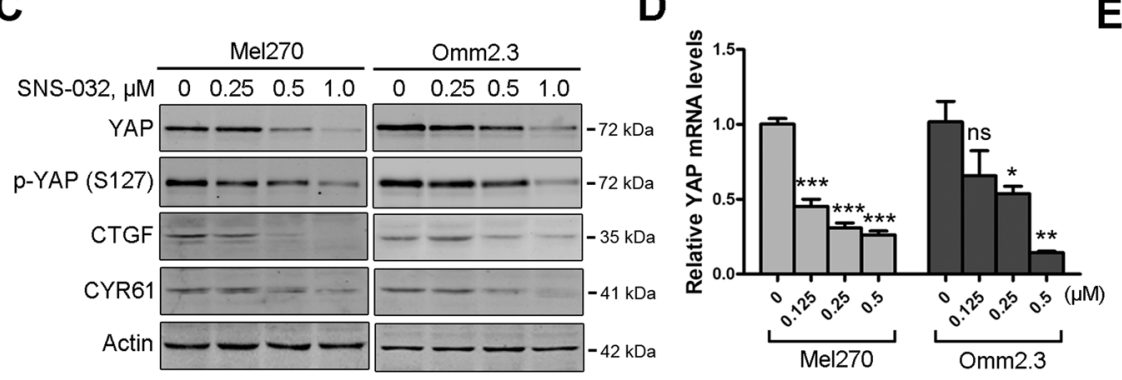

E

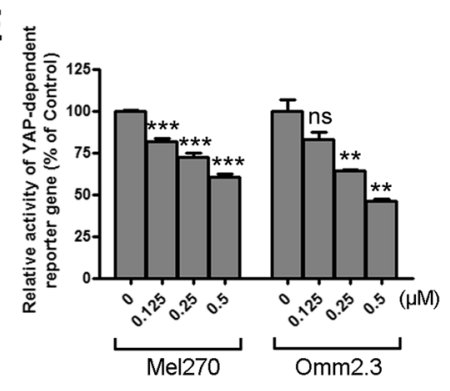

$\mathrm{F}$
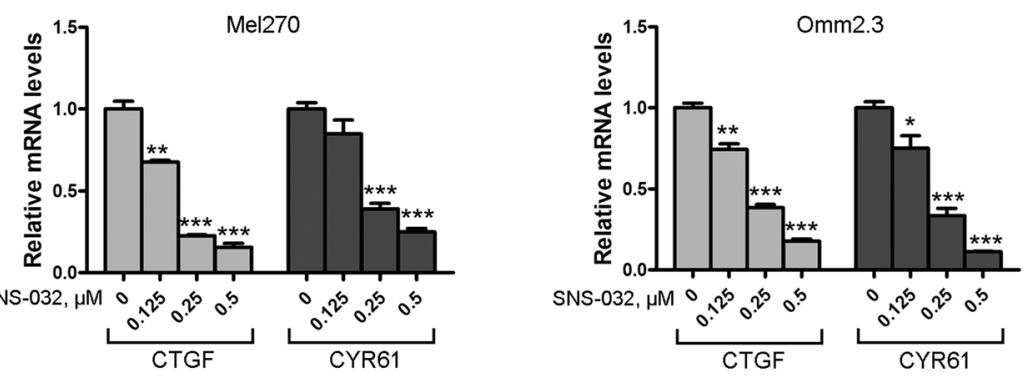

G

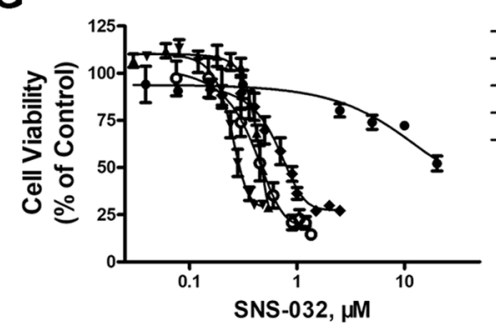

I

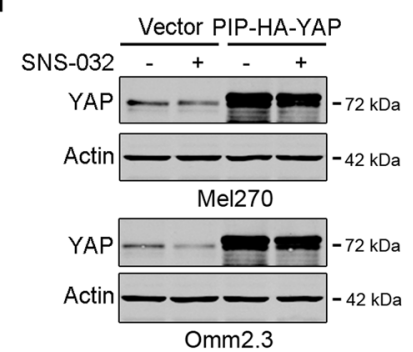

Omm2.3

H
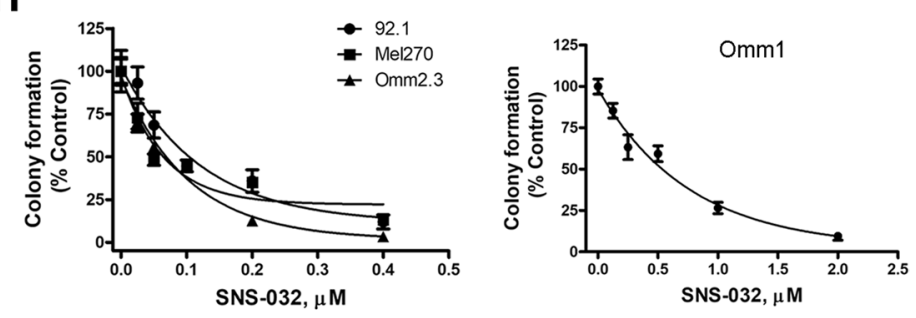

J
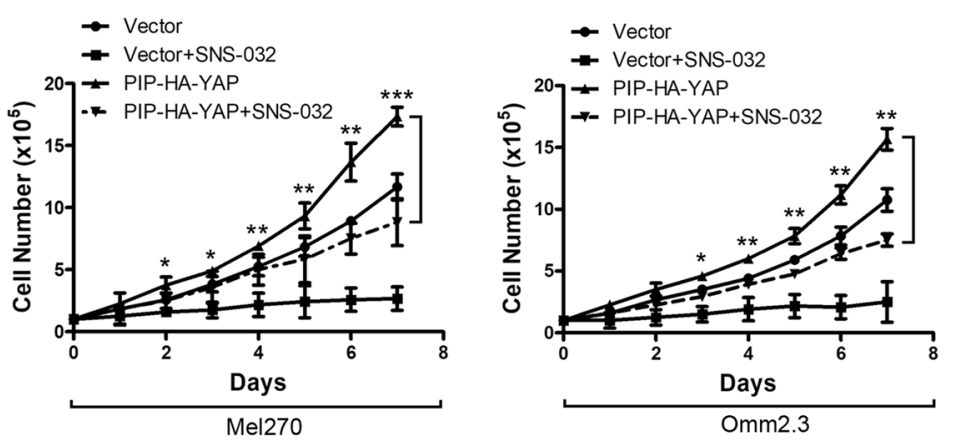

Fig. 1 (See legend on previous page.) 
(See figure on next page.)

Fig. 2 SNS-032 induces apoptosis in human UM cells. a and $\mathbf{b}$ UM cells were incubated with increasing concentrations of SNS-032 for $48 \mathrm{~h}$, apoptosis was determined by flow cytometry after Annexin V-FITC/PI dual staining. a Representative flow dot plots for Omm2.3 are shown; $\mathbf{b}$ Quantitative analysis from three independent experiments. Data represent mean $\pm \mathrm{SEM} .{ }^{*}, P<0.05 ;{ }^{* *}, P<0.01$; ${ }^{* *}, P<0.001$, one-way ANOVA, post hoc intergroup comparisons, Tukey's test. c Dose-dependent cleavage of PARP and activation of caspase-3 were detected by Western blot after exposure with SNS-032 for $48 \mathrm{~h}$ in UM cells. $\mathbf{d}$ Western blot analysis of levels of cytochrome $\mathrm{c}$ in the cytosolic fractionations was performed in UM cells treated with SNS-032 (1.0 $\mu \mathrm{M})$ for the indicated durations. The cytosolic fractionations were not contaminated as indicated by a mitochondria marker COX II. e SNS-032 induced mitochondrial membrane depolarization in UM cells. 92.1 and Omm2.3 cells incubated with SNS-032 were stained with CMXRos and MTGreen, and then analyzed by flow cytometry. Results for three independent experiments are shown. ${ }^{* * *}, P<0.001$, one-way ANOVA, post hoc intergroup comparisons, Tukey's test. $\mathbf{f}$ Western blot analysis of apoptosis-related proteins expression was performed in UM cells treated with SNS-032. g SNS-032 dose-dependently decreased mRNA levels of BIRC5 gene (encoding Survivin) in UM cells. *, $P<0.05$; ${ }^{* *}, P<0.01 ; * * *, P<0.001$, one-way ANOVA, post hoc intergroup comparisons, Tukey's test. $\mathbf{h}$ and $\mathbf{i}$ Overexpression of Survivin abrogated whereas knockdown of Survivin potentiated the SNS-032-induced apoptosis. $\mathbf{h}$ Omm1 cells were transduced with lentiviral Vector (pTSB) or Survivin (pTSB-Survivin) constructs for $48 \mathrm{~h}$, then treated with SNS-032 for $24 \mathrm{~h}$; i 92.1 cells were transduced with lentiviral Vector (Scramble) or Survivin shRNAs (shSurvivin \#1 and \#2) constructs for $48 \mathrm{~h}$, then treated with SNS-032 for $24 \mathrm{~h}$. The protein levels of PARP and Survivin were detected by Western blot analysis (top); cell death was examined by trypan blue exclusion assay (bottom). ${ }^{*}, P<0.01 ;{ }^{* *}, P<0.001$, Student's $t$ test 
A
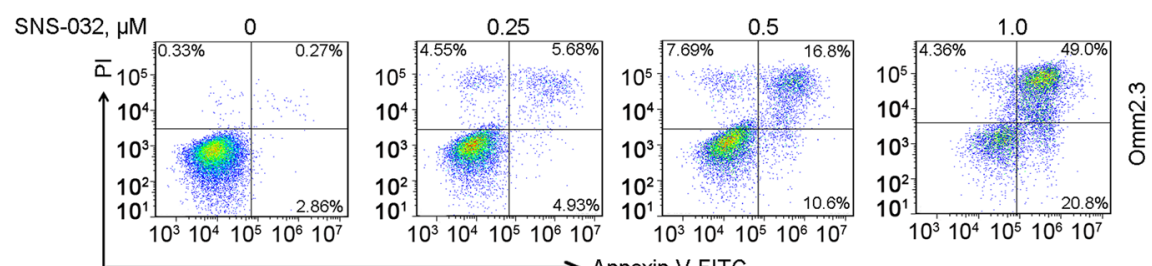

B

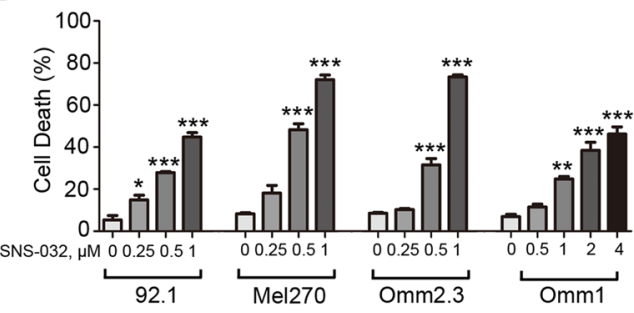

D

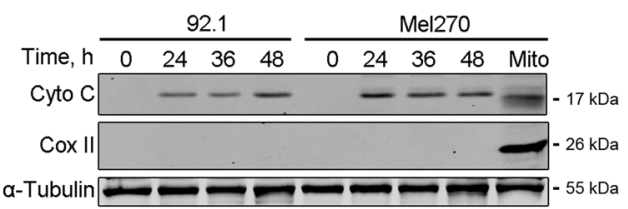

$\mathbf{F}$

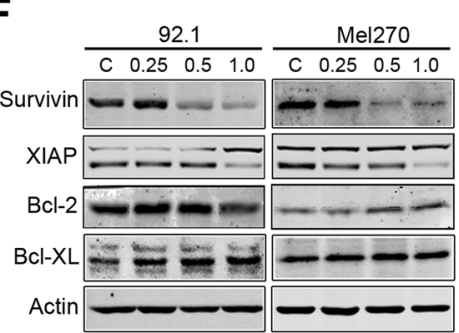

H

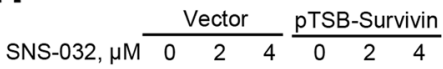
PARP Survivin - $-17 \mathrm{kDa}$ Actin $\square-42 \mathrm{kDa}$

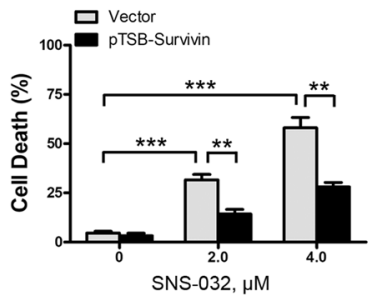

C

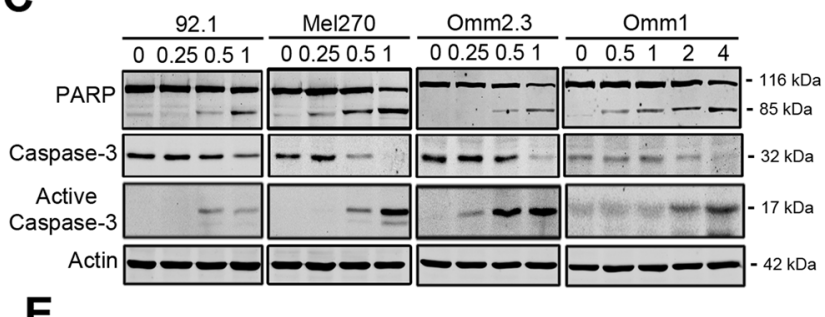

E
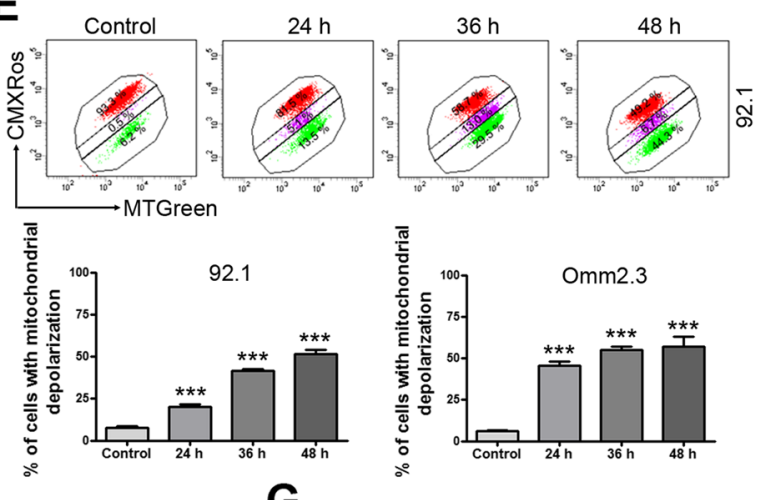

$\mathbf{G}$

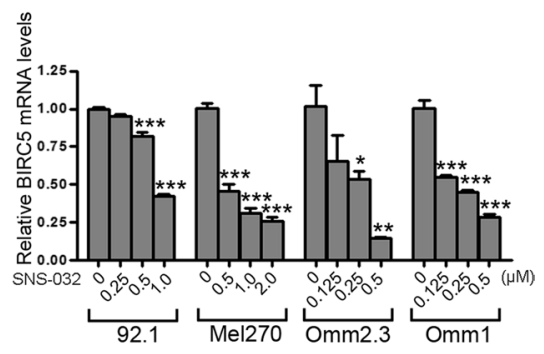

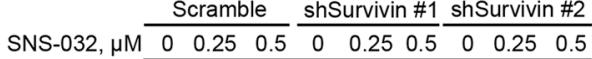
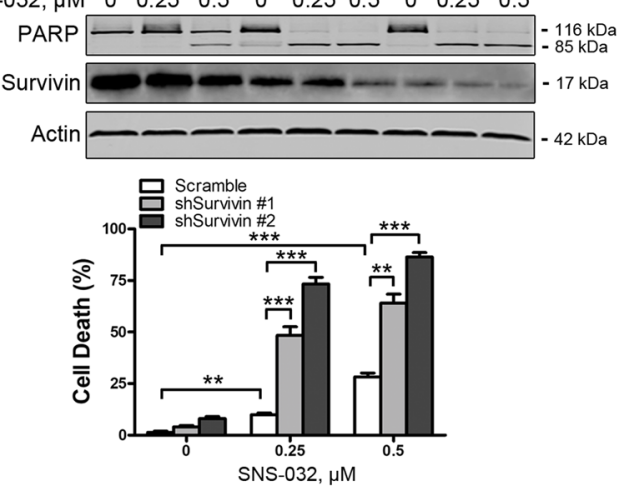

Fig. 2 (See legend on previous page.) 


\section{(See figure on next page.)}

Fig. 4 SNS-032 impairs properties of cancer stem-like cells through lowering transcription factor KLF4 in UM cells. a SNS-032 suppressed melanosphere growth and serially replating capacity in UM cells. Twenty-four hours after treatment with SNS-032 (0.25 $\mu$ M), Mel270 and Omm2.3 cells were harvested, suspended in stem cell culture medium, and plated in ultra-low attachment 24-well plates (1000 cells/well). Melanospheres were counted on day 7. The cells were then harvested and performed for the secondary and tertiary rounds of melanosphere culture, respectively. ${ }^{*}, P<0.05 ;{ }^{* *}, P<0.01 ;{ }^{* *}, P<0.001$, Student's $t$ test. b SNS-032 decreased ALDH ${ }^{+}$cells in UM cells. Mel270 and Omm2.3 cells were treated with SNS-032 for $48 \mathrm{~h}$, the percentage of $\mathrm{ALDH}^{+}$cells was detected by flow cytometry. Quantitive analysis of $\mathrm{ALDH}^{+}$cells from three independent experiments is shown. ${ }^{* *}, P<0.001$, Student's $t$ test. c SNS-032 reduced the frequency of CSCs in UM performed by limiting dilution assay in NOD-SCID mice. Representative images of tumor removed from the mice of each group are shown (left). The frequency of CSCs in UM is shown (right). d SNS-032 decreased the protein levels of KLF4. UM cells were exposed to SNS-032 for $48 \mathrm{~h}$, the levels of stemness-related proteins were detected by Western blot analysis. e SNS-032 inhibited the gene transcription of KLF4. The mRNA levels of KLF4 were measured after $24 \mathrm{~h}$ exposure to SNS-032 by qRT-PCR, and expressed as relative levels compared with controls. ${ }^{*}, P<0.05 ;{ }^{*}, P<0.01 ; * * *, P<0.0001$, one-way ANOVA, post hoc intergroup comparisons, Tukey's test. $\mathbf{f}-\mathbf{h}$ Ectopic expression of KLF4 attenuated SNS-032-mediated decrease of CSCs properties in UM cells. $\mathbf{f}$ The protein levels of KLF4 were examined by Western blot analysis after Mel270 cells stably transduced with lentiviral vector or construct encoding human KLF4. $\mathbf{g}$ Ectopic expression of KLF4 increases self-renewal capacity evaluated by melanosphere growth and serially replating assay. $\mathbf{h}$ Overexpression of KLF4 increases the percentage of $\mathrm{ALDH}^{+}$cells detected by flow cytometry. i-k Silencing KLF4 potentiated SNS-032-mediated decrease of CSCs properties in UM cells. i The protein levels of KLF4 were examined by Western blot analysis after Mel270 cells stably transduced with lentiviral vector or shRNAs against human KLF4. j Knockdown of KLF4 decreased melanosphere growth and serially replating capacity. $\mathbf{k}$ Knockdown of KLF4 reduced the percentage of $\mathrm{ALDH}^{+}$cells. ${ }^{*}, P<0.05 ;{ }^{* *}, P<0.01 ;{ }^{* *}, P<0.001$, Student's $t$ test 

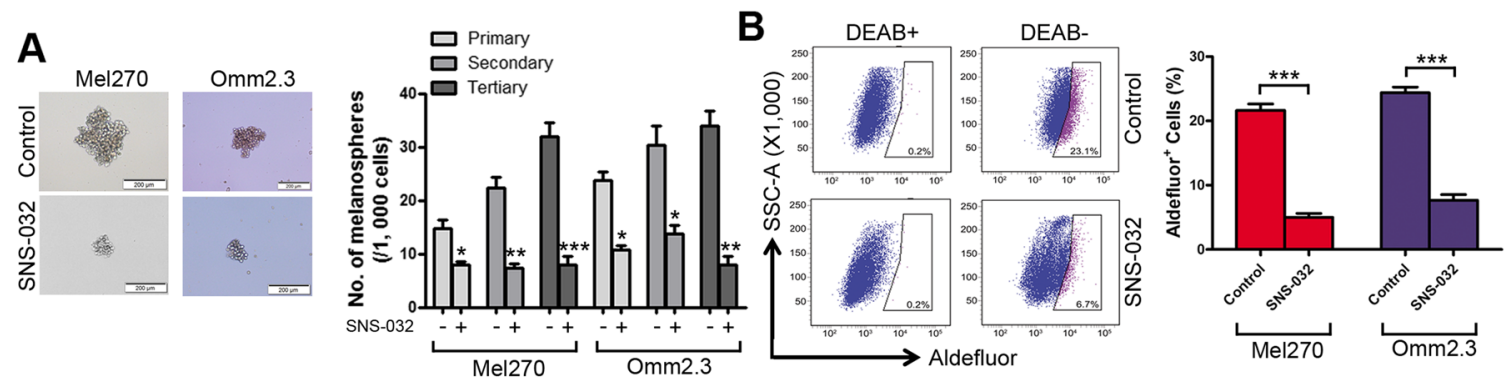

C

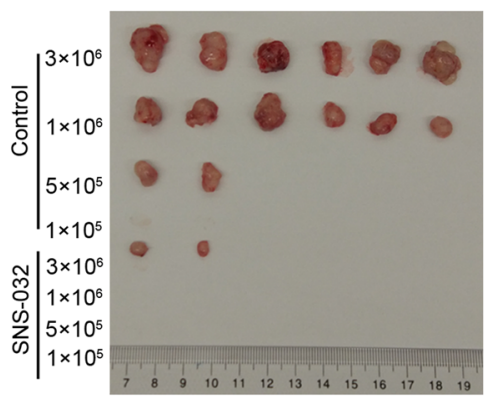

D
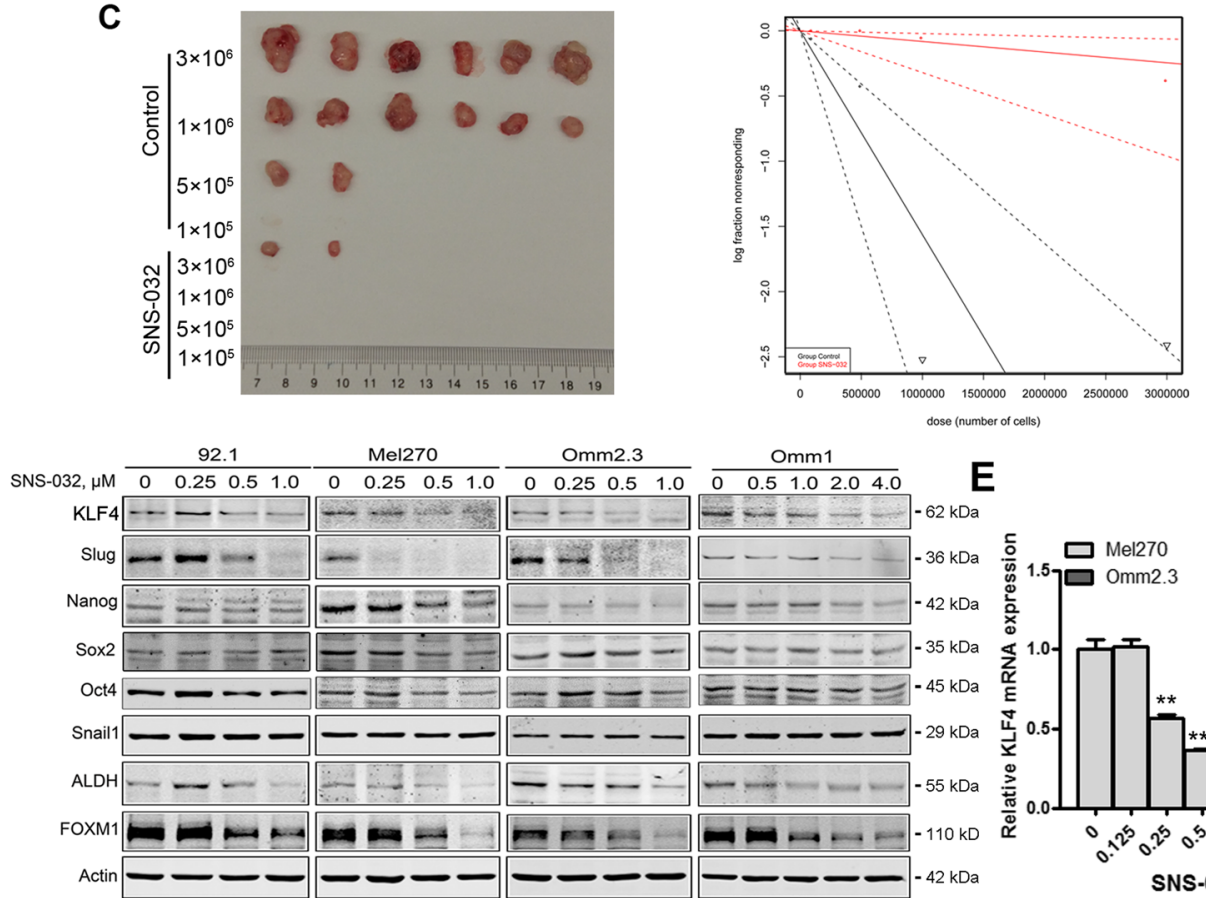

dose (number of cells)

$\mathbf{F}$

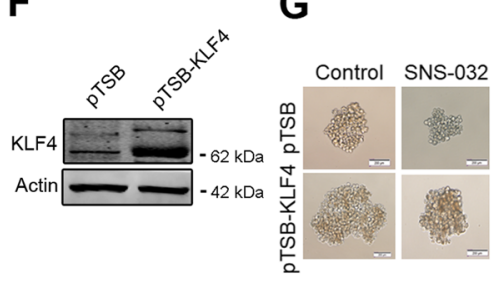

I
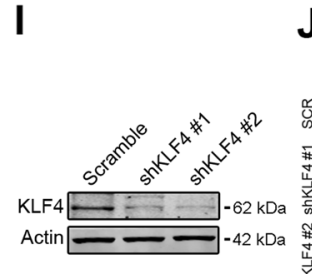

J
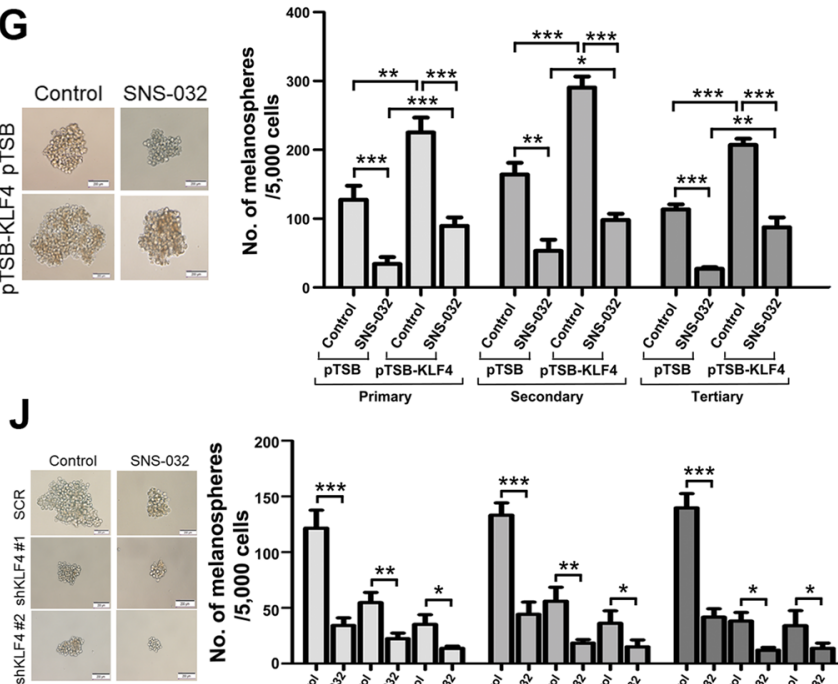

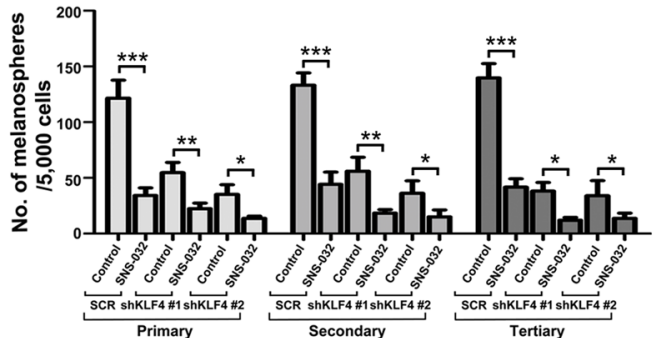

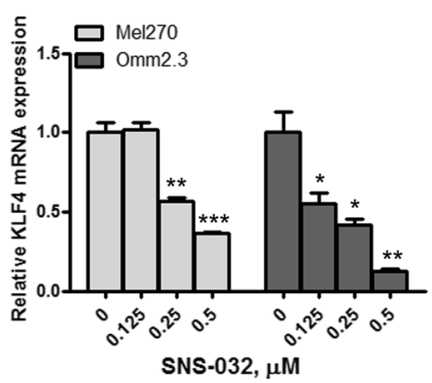

H

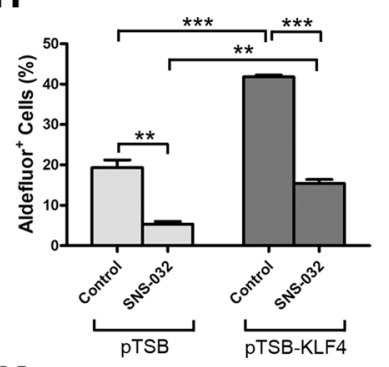

K

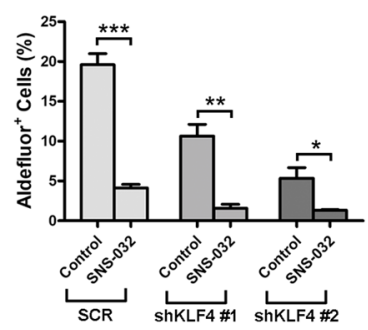

Fig. 4 (See legend on previous page.) 